\title{
A COMPREHENSIVE ANALYSIS OF DISTRACTED DRIVING USING A DRIVING SIMULATOR
}

\author{
Samira Ahangari ${ }^{1}$, Mansoureh Jeihani ${ }^{2}$, Behrouz Salahshour $^{3}$, Martin Ndegwa $^{4}$ \\ 1,2,4 Department of Transportation and Urban Infrastructure Studies, Morgan State University, \\ Baltimore, MD 21251, USA \\ ${ }^{3}$ Department of Civil Engineering, Old Dominion University, Norfolk, VA 23529, USA
}

Received 26 February 2020; accepted 31 March 2020

\begin{abstract}
Studies have examined the detrimental impact of distracted driving on safety; however, the effect of different types of distraction accompanied by different road classes has not been investigated. This study used a high-fidelity driving simulator to examine the driving behavior of young participants while engaged in various distractions - no cell phone, hands-free call, hand-held call, voice commands text, text, clothing, eating or drinking - on different road classes: rural collector, freeway, urban arterial, and local road in a school zone. Some 92 participants drove a simulated network in Baltimore County with seven scenarios (one base scenario without any distraction and six different types of distraction). Participants also completed questionnaires documenting demographics and driving behavior before and after the driving simulator experience. The descriptive and statistical analysis revealed the negative impact of distraction on safety, such that participants exhibited greater fluctuations in speed, changed lanes significantly more times, and deviated from the center of the road when they were distracted while driving. The results indicated that drivers reduced their speed up to $33 \%$ while distracted with hands-free/voice command cell phone usage, which is inconsistent with the current cell phone usage policies in most states.
\end{abstract}

Keywords: distracted driving, driver behavior, driving simulator.

\section{Introduction}

Distracted drivers are involved in about $9 \%$ of all crash fatalities, accounting for 3,166 deaths including 497 pedestrians in 2017 (Neale et al., 2005). With the prevalence of cell phones and their various uses, these numbers may potentially arise. Therefore, more in-depth knowledge of accepted safe driving behaviors is needed.

Driving safely consists of performing a collection of visual-motor tasks involving a vehicle and everything else in which the tasks vary as a function of time, place and speed (Lee, 2017). Driver distraction occurs when a driver "is delayed in recognition of information needed to safely accomplish the driving task because some event, activity, object or person within or outside the vehicle compelled or tended to include the driver's shifting attention away from the driving task," and is the major cause of driver inattention (Stutts et al., 2001). Stutts et al., simply being "lost in thought" is another category of inattention which is distinguished from extrinsic distraction.

\footnotetext{
${ }^{1}$ Corresponding author: saaha1@morgan.edu
} 
Driver distractions can be further divided into driving environment complexity effects, such as roadside advertisements, and invehicle effects including talking with other passengers, eating/drinking, radio tuning, or more recently cell phone usage (Horberry et al., 2006). Some researchers use tuning the radio as a benchmark for distracted driving (Lee et al., 2018). Numerous studies show that cell phone usage compromises drivers' attention (Charlton, 2009; Choudhary \& Velaga, 2017; Drews et al., 2004; Lipovac et al., 2017; Overton et al., 2015; Schlehofer et al., 2010, Ahangari et al, 2019). However, not all usages of the phone have similar distracting effects. Texting has been found to be more distracting (Drews et al., 2009; Gliklich et al., 2016), perhaps because it has both a cognitive demand and a physical constraint compared to talking on the phone (Stavrinos et al., 2013). However, other studies suggest that texting, even using text-to-speech technology, still impairs drivers' reaction time and attention span (He et al., 2014; NHTSA, 2015).

In a report published by AT\&T, about $97 \%$ of teenagers admitted knowing the dangers of texting and driving; however, $43 \%$ reported that they still text sometimes. About $75 \%$ of the respondents have described texting or emailing while driving as "common" among their friends and peers. More than $90 \%$ of the participants agreed that a severe legal action (license suspension or a $\$ 500$ ticket) would be the most effective preventive method (Tomas et al., 2012). The results from similar penalties support these survey findings. For instance, (Liu et al., 2019) have investigated the effectiveness of California's 2008 ban on hand-held phones while driving. Their results show the effectiveness of these regulations and support a full ban on cell phone usage, not just hand-held devices (Liu et al., 2019).
Other researchers explored the willingness of drivers to use applications that limit some phone usage such as texting but allow access to other applications like GPS in order to reduce exposure to high-risk behaviors while driving (Oviedo-Trespalacios et al., 2019).

The three types of research involving cell phones and other driving distractions are: epidemiological studies, field studies, and recent research conducted using simulators (Lipovac et al., 2017). (Törnros \& Bolling, 2005) investigated the effects of hand-held and hands-free phones on driving performance using a simulator and found that while hands-free usage of a phone improved lateral driving control during the conversation period, distraction measurements during dialing and other activities were no different when compared to hand-held usage (Törnros \& Bolling, 2005). Another study compared talking on a cell phone to talking to a passenger and found that phone conversations caused a greater deceleration in response time and thereby posed a higher collision hazard (Charlton, 2009). Lateral performance measures during distracted driving were investigated by (Choudhary et al., 2017) on 100 drivers and the results indicated a significant decrease in performance during the texting and driving task. They suggest in-vehicle monitoring devices for driver distraction measurements (Choudhary \& Velaga, 2017).

In the current study, a simulator is used to investigate the effects of six different scenarios of in-vehicle distractions including usage of a cell phone with and without handsfree capability on different types of roads (rural collector, freeway, urban arterial, and local road in a school zone). Drivers were given a survey before and after their driving experience. The goal of this research 
is to investigate the driver's behavior in the presence of different types of distraction on different types of roads and compare it with no distraction.

\section{Methods}

\subsection{Participants}

We recruited 92 young participants from Morgan State University and the Baltimore metro area via flyers distributed manually, online and through social media. Flyer content included contact information, a summary of the requirements for the study, and an explanation of the monetary compensation for driving the simulator. Subsequently, we screened prospective participants for eligibility and scheduled them to drive in the simulator environment.

Participants were required to possess a valid driver's license and were compensated at $\$ 15$ per hour for their study participation. In addition, participants were asked to use their own cellphone during the driving experience and bring a hands-free device and a jacket/sweater with them for different distracting experiences. We provided them with water and candy for drinking and eating distractions.

\subsection{Procedure}

Under the supervision of an advisor, a team of undergraduate and graduate student research assistants observed the driving tasks. Participants were asked to fill out a pre-survey questionnaire, then drive for about two hours in different simulated scenarios, and then fill out the post-survey questionnaire after driving to find the effect of their experience on driver behavior.

\subsection{Driving Simulator}

The observer made sure that the participants' cell phones worked properly. They instructed the participants to drive briefly to familiarize themselves with the simulator environment and explained the procedure before each scenario. Participants were instructed to adjust their cell phone to a loud ringer volume and have it handy before beginning each scenario.

The participants started driving in a base scenario with no distraction to compare that driving behavior with other types of distraction. Participants then drove six different distraction scenarios in no particular order - including hands-free call, hand-held call, voice commands text, text, clothing, and eating or drinking - on a road network north of Baltimore that includes four different classes of the road (rural collector, freeway, urban arterial, and local road in a school zone) with different numbers of lanes and speed limits for each road (Figure 1).

There was one type of distraction in each scenario and the distraction happened exactly at the same location. The questions involved were similar in cognitive load (but different in content) for a fair comparison between different distractions. Participants were instructed to answer a phone call, respond to a text message upon receiving it, clothing, and drink or eat during the simulated drive. Participants did not know the questions they would receive as a call or text during any given scenario so that they would not exhibit anticipatory behavior that would have influenced their driving behavior.

During each driving scenario, participants were instructed to drive as they typically 
would on a real road for approximately 15 min and comply with the speed limit. The virtual roads environment featured one lane with a $30 \mathrm{mph}$ speed limit for the rural collector, three lanes with $55 \mathrm{mph}$ for the freeway, two lanes with $45 \mathrm{mph}$ for the urban arterial, and one lane with $30 \mathrm{mph}$ for the local road. The daytime scenery closely matched driving situations in the Baltimore metropolitan area. Traffic flow and density were the same in all seven scenarios. The driving experience in each scenario progresses from rural to freeway, then to urban and finally to a local road, and participants received the distraction in the same location in each scenario (Figure 1).

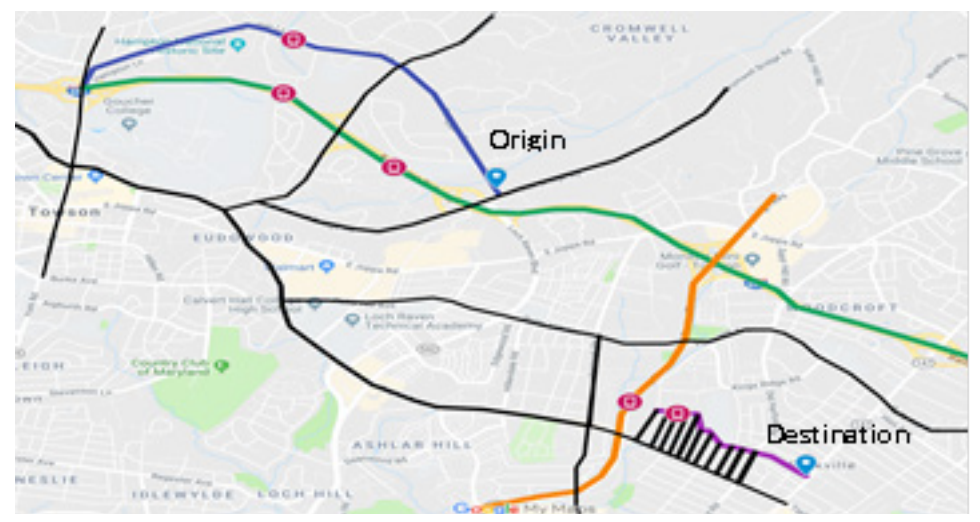

Fig. 1.

Study Network

\subsection{Text and Call Condition}

The observers used a script that required participants to respond to various openended questions. Typical questions were "What comes to your mind when you hear the word 'America'?" or "What's your number one vacation destination?" and "How many of your friends have names beginning with 'F?". The participants were distracted five times during each scenario including once in a rural area, twice on the freeway, and once in the urban area and in the local area at exactly the same position.

\subsection{Questionnaires}

The questionnaires involved completing demographic information and questions about real driving behavior before the driving simulator experience (pre-survey) and driving behavior after driving the simulator (post-survey). Observers gave participants the option of completing the questionnaire on their own or with the assistance of the observer.

\subsection{Driving Simulator}

Participants drove about 10 miles in each scenario in a high-fidelity driving simulator to provide a measure of driving performance under different distracting tasks (http:// www.forum8.co.jp). The simulation was displayed on three, 40-inch LCD screens. Participants sat within the simulator's driver compartment, which provided a view of the roadway and dashboard instruments including 
a speedometer (Figure 2). The vehicle was controlled by moving a steering wheel in the typical fashion and pressing the accelerator and brake pedals accordingly. Naturalistic engine sounds, road noise, and sounds of passing traffic simulated the real world.

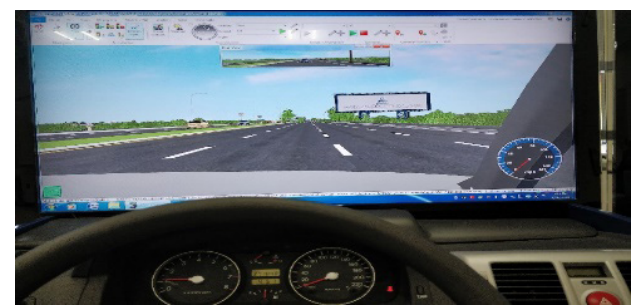

Fig. 2.

Driving Simulator

\subsection{Driving Performance}

Different information about the driver's behavior including speed, throttle, brake, steering velocity, offset from road center, and lane change was calculated for the distraction condition. For example, we calculated offset from the road center, which was reported as the deviated distance from the road center toward the right or left side, and saved it as an indicator of impaired driving performance. Greater within-lane deviation indicated poorer driving precision. Average driving speed within the distraction area (the distraction area was different for each road) was calculated based on the speed of the vehicle and time of distraction and computed as the degree to which drivers
Simulated vehicles with varying speed and volume were programmed to interact with the participant driver. Researchers could safely assess the impact of distracted driving by comparing drivers' behavior under different types of distraction with no distraction.

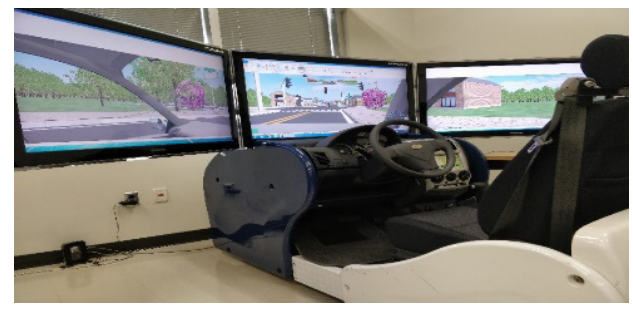

changed their speed for each scenario. We used lane change frequency, defined as the number of times the driver changed lanes, as an indicator. The brake and throttle behavior indicates distraction, which compares with no distraction behavior. The severe force of a brake demonstrates inattention to the road and taking the mind off the road.

\subsection{Data Analyzing}

Descriptive statistics were obtained from presurvey questionnaire data regarding participant characteristics. Some $56.52 \%$ of participants were male and $43.48 \%$ were female. The age group of participants was between 18 to 40 years old; $44.57 \%$ of which were in the age group of 21 to 25 years (Table 1 ). 


\section{Table 1}

Sociodemographic Analysis

\begin{tabular}{|c|c|c|c|}
\hline \multicolumn{2}{|c|}{ Variable } & \multirow{2}{*}{$\frac{\text { Frequency }}{40}$} & \multirow{2}{*}{$\frac{\text { Percent }}{43.48}$} \\
\hline & Female & & \\
\hline actiot & Male & 52 & 56.52 \\
\hline \multirow{5}{*}{ Age } & 18 to 20 & 15 & 16.30 \\
\hline & 21 to 25 & 41 & 44.57 \\
\hline & 26 to 30 & 15 & 16.30 \\
\hline & 31 to 35 & 9 & 9.78 \\
\hline & 36 to 40 & 12 & 13.04 \\
\hline \multirow{5}{*}{ Education Status } & Associate degree & 7 & 7.61 \\
\hline & College graduate & 14 & 15.22 \\
\hline & College student & 50 & 54.35 \\
\hline & High School or less & 15 & 16.30 \\
\hline & Postgraduate & 6 & 6.52 \\
\hline \multirow{4}{*}{ Employment Status } & No & 44 & 47.83 \\
\hline & Full time & 18 & 19.57 \\
\hline & Part-time & 30 & 32.61 \\
\hline & Total & 92 & 100.00 \\
\hline \multirow{6}{*}{$\begin{array}{l}\text { Household Annual } \\
\text { Income }\end{array}$} & $\$ 20 \mathrm{~K}$ to $\$ 30 \mathrm{~K}$ & 18 & 19.57 \\
\hline & $\$ 30 \mathrm{~K}$ to $\$ 50 \mathrm{~K}$ & 19 & 20.65 \\
\hline & $\$ 50 \mathrm{~K}$ to $\$ 75 \mathrm{~K}$ & 11 & 11.96 \\
\hline & $\$ 75 \mathrm{~K}$ to $\$ 100 \mathrm{~K}$ & 2 & 2.17 \\
\hline & Less than $\$ 20 \mathrm{~K}$ & 27 & 29.35 \\
\hline & More than $\$ 100 \mathrm{~K}$ & 15 & 16.30 \\
\hline \multirow{4}{*}{ Household Size } & 1 & 23 & 25.00 \\
\hline & 2 & 23 & 25.00 \\
\hline & 3 & 18 & 19.57 \\
\hline & an or more & 28 & 30.43 \\
\hline
\end{tabular}

\section{Results and Discussion}

\subsection{Pre-survey Analyzing}

The pre-survey questionnaire demonstrated that $43.5 \%$ of participants use the hands-free phone, $22.8 \%$ use a hand-held phone, $21.7 \%$ text, $8.7 \%$ read social media, $6.5 \%$ read email, $16.3 \%$ take pictures, $45.7 \%$ drink or eat, and $1.1 \%$ change their clothes when driving (Figure 3). 


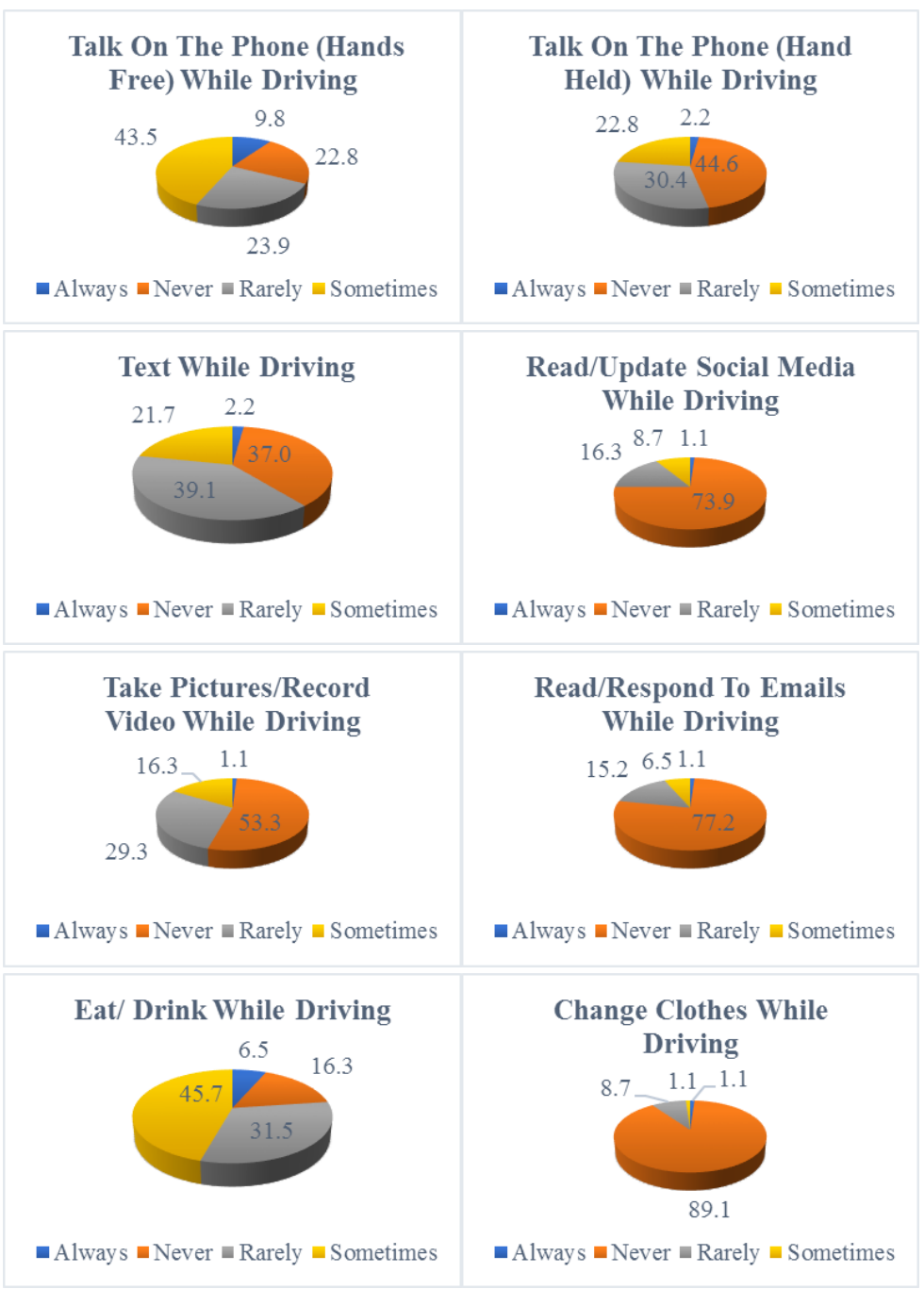

Fig. 3.

Pre Survey Analysis

\subsection{Post-survey Analyzing}

The results of the post-survey questionnaire show a great change in the attitude of drivers after being involved in such a study. Some $36.5 \%$ of the participants stated that the driving simulator experience encouraged them to reduce cell phone use while driving.
And $47.2 \%$ were doubtful about using technologies while driving for safety's sake compared to only $15.2 \%$ who had doubts in the pre-survey questionnaire. After driving, $51.8 \%$ expressed doubt about their ability to use cell phones freely and not make any driving mistakes; $26 \%$ had stated they were doubtful in pre-survey (Figure 4). 
To what extent are you confident that YOU, driving in the following situations, would NOT experience any driving mistakes such as deviating from the destination, going through a red light, near-crash experience, crash, etc.? [Technologies such as voice to text]

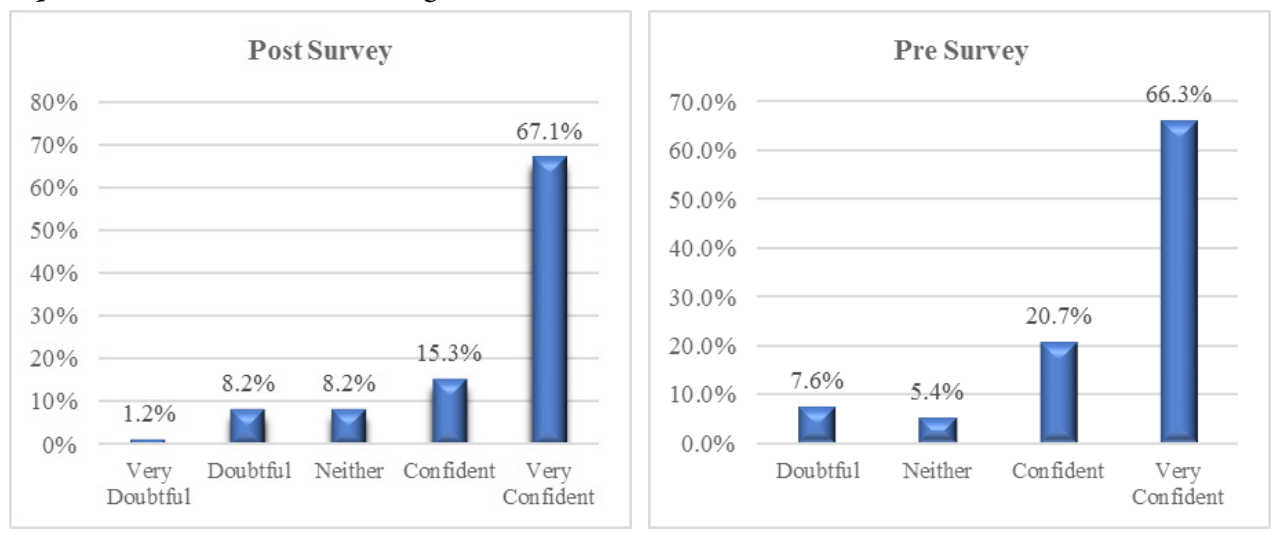

To what extent are you confident that YOU, driving in the following situations, would NOT experience any driving mistakes such as deviating from the destination, going through a red light, near-crash experience, crash, etc.? [No cell phone while driving]
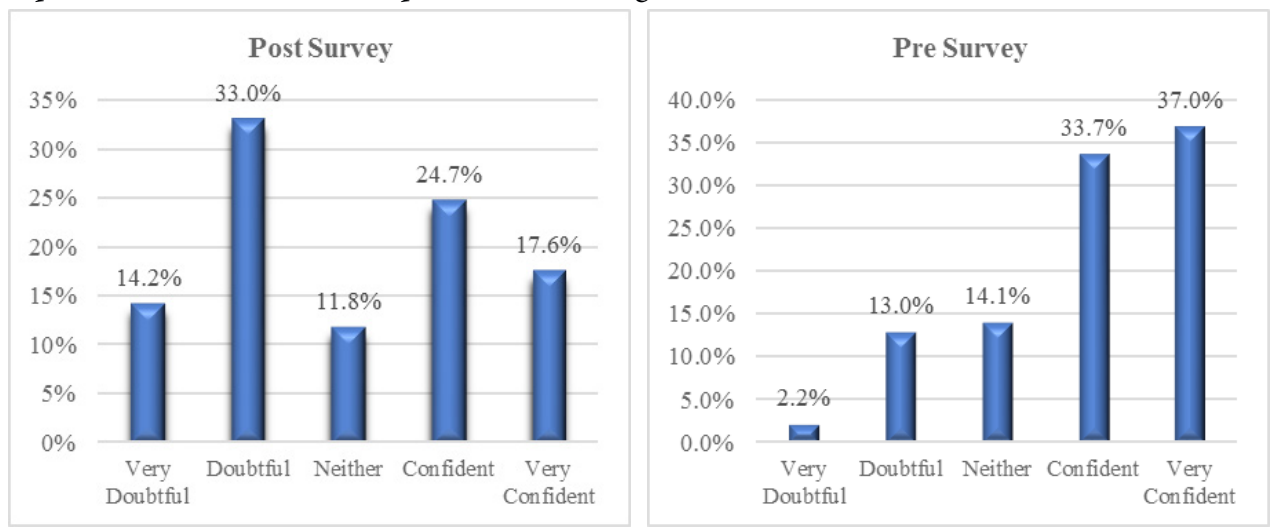

Fig. 4.

Comparison of Pre and Post Survey Results

\subsection{Driving Simulator Analyzing}

We conducted several ANOVA to compare the driving behavior (speed, throttle, brake, steering velocity, offset from road center, and lane change) under different types of distractions (no distraction, hands-free call, hands-held call, voice commands text,

text, taking on or off clothes, and eating or drinking) and considering different road classes. The results (Table 2 ) revealed significant differences in speed, throttle, brake, steering velocity, offset from road center, and lane change when comparing different types of distraction to no distraction. 
Table 2

Descriptive and ANOVA Analysis

\begin{tabular}{|c|c|c|c|c|c|c|c|c|c|c|c|c|c|c|c|c|c|}
\hline \multicolumn{2}{|c|}{ Variables } & $\mathbf{N}$ & Mean & $\begin{array}{c}\text { Std. } \\
\text { Devia- } \\
\text { tion }\end{array}$ & $\mathbf{F}$ & $\mathbf{N}$ & Mean & $\begin{array}{c}\text { Std. } \\
\text { Devia- } \\
\text { tion }\end{array}$ & $\mathbf{F}$ & $\mathbf{N}$ & Mean & $\begin{array}{c}\text { Std. } \\
\text { Devia- } \\
\text { tion }\end{array}$ & $\mathbf{F}$ & $\mathbf{N}$ & Mean & $\begin{array}{c}\text { Std. } \\
\text { Devia- } \\
\text { tion }\end{array}$ & $\mathbf{F}$ \\
\hline \multirow{2}{*}{ DV } & \multirow{2}{*}{$\begin{array}{l}\text { IV (Type of } \\
\text { Distraction) }\end{array}$} & \multicolumn{16}{|c|}{ Type of Road } \\
\hline & & \multicolumn{4}{|c|}{ Rural Collector } & \multicolumn{4}{|c|}{ Freeway } & \multicolumn{4}{|c|}{ Urban Arterial } & \multicolumn{4}{|c|}{ Local Road in a School Zone } \\
\hline \multirow{7}{*}{ Speed } & $\begin{array}{c}\text { No } \\
\text { Distraction }\end{array}$ & 78 & 70.31 & 19.15 & \multirow{7}{*}{$10.28^{*}$} & 156 & 83.16 & 9.67 & \multirow{7}{*}{$34.45^{*}$} & 78 & 52.22 & 14.55 & & 63 & 35.72 & 11.32 & \multirow{7}{*}{$11.72^{*}$} \\
\hline & $\begin{array}{c}\text { Hands-Free } \\
\text { Call }\end{array}$ & 81 & 55.41 & 14.40 & & 162 & 74.24 & 8.25 & & 81 & 44.85 & 7.38 & & 64 & 27.75 & 10.82 & \\
\hline & $\begin{array}{c}\text { Hand-Held } \\
\text { Call }\end{array}$ & 81 & 55.00 & 14.94 & & 160 & 74.18 & 7.09 & & 79 & 45.34 & 8.55 & & 70 & 26.29 & 8.98 & \\
\hline & $\begin{array}{c}\text { Voice } \\
\text { Commands } \\
\text { Text }\end{array}$ & 78 & 56.62 & 14.63 & & 154 & 73.80 & 8.10 & & 77 & 43.32 & 9.77 & $6.68^{*}$ & 69 & 23.78 & 10.68 & \\
\hline & Text & 77 & 60.07 & 13.95 & & 154 & 73.35 & 8.37 & & 77 & 45.16 & 9.65 & & 70 & 25.30 & 9.64 & \\
\hline & Clothing & 42 & 53.66 & 16.60 & & 84 & 69.78 & 6.99 & & 42 & 44.34 & 9.44 & & 38 & 17.96 & 11.31 & \\
\hline & $\begin{array}{l}\text { Eating or } \\
\text { Drinking }\end{array}$ & 43 & 58.45 & 15.47 & & 86 & 70.35 & 7.00 & & 43 & 42.20 & 10.28 & & 40 & 26.14 & 11.54 & \\
\hline \multirow{7}{*}{ Throttle } & $\begin{array}{c}\text { No } \\
\text { Distraction }\end{array}$ & 78 & 0.37 & 0.19 & & 156 & 0.33 & 0.15 & \multirow{7}{*}{$13.01^{*}$} & 78 & 0.29 & 0.16 & & 66 & 0.12 & 0.07 & \multirow{7}{*}{$2.59^{*}$} \\
\hline & $\begin{array}{c}\text { Hands-Free } \\
\text { Call }\end{array}$ & 81 & 0.25 & 0.12 & & 162 & 0.29 & 0.13 & & 81 & 0.23 & 0.10 & & 64 & 0.10 & 0.07 & \\
\hline & $\begin{array}{c}\text { Hand-Held } \\
\text { Call }\end{array}$ & 81 & 0.27 & 0.15 & & 160 & 0.29 & 0.13 & & 79 & 0.24 & 0.11 & & 70 & 0.11 & 0.08 & \\
\hline & $\begin{array}{c}\text { Voice } \\
\text { Commands } \\
\text { Text }\end{array}$ & 78 & 0.26 & 0.12 & $8.45^{*}$ & 154 & 0.29 & 0.12 & & 77 & 0.25 & 0.10 & 1.83 & 69 & 0.09 & 0.06 & \\
\hline & Text & 77 & 0.33 & 0.15 & & 154 & 0.30 & 0.15 & & 77 & 0.27 & 0.15 & & 70 & 0.11 & 0.05 & \\
\hline & $\begin{array}{l}\text { Taking off or } \\
\text { on Clothing }\end{array}$ & 42 & 0.38 & 0.15 & & 84 & 0.26 & 0.11 & & 42 & 0.25 & 0.15 & & 38 & 0.12 & 0.06 & \\
\hline & $\begin{array}{l}\text { Eating or } \\
\text { Drinking } \\
\end{array}$ & 43 & 0.39 & 0.19 & & 86 & 0.28 & 0.13 & & 43 & 0.26 & 0.16 & & 40 & 0.12 & 0.07 & \\
\hline \multirow{7}{*}{ Brake } & $\begin{array}{c}\text { No } \\
\text { Distraction }\end{array}$ & 78 & 0.01 & 0.01 & & 156 & 0.01 & 0.01 & \multirow{7}{*}{$3.50^{*}$} & 78 & 0.01 & 0.01 & & 66 & 0.10 & 0.09 & \multirow{7}{*}{2.09} \\
\hline & $\begin{array}{c}\text { Hands-Free } \\
\text { Call }\end{array}$ & 81 & 0.00 & 0.01 & & 162 & 0.01 & 0.01 & & 81 & 0.01 & 0.01 & & 64 & 0.10 & 0.10 & \\
\hline & $\begin{array}{c}\text { Hand-Held } \\
\text { Call }\end{array}$ & 81 & 0.01 & 0.01 & & 160 & 0.01 & 0.01 & & 79 & 0.01 & 0.01 & & 70 & 0.09 & 0.08 & \\
\hline & $\begin{array}{c}\text { Voice } \\
\text { Commands } \\
\text { Text }\end{array}$ & 78 & 0.00 & 0.01 & $2.01^{*}$ & 154 & 0.01 & 0.01 & & 77 & 0.01 & 0.01 & 0.63 & 69 & 0.09 & 0.07 & \\
\hline & Text & 77 & 0.00 & 0.01 & & 154 & 0.01 & 0.01 & & 77 & 0.01 & 0.01 & & 70 & 0.09 & 0.07 & \\
\hline & Clothing & 42 & 0.01 & 0.02 & & 84 & 0.01 & 0.01 & & 42 & 0.01 & 0.01 & & 38 & 0.12 & 0.09 & \\
\hline & $\begin{array}{l}\text { Eating or } \\
\text { Drinking }\end{array}$ & 43 & 0.00 & 0.01 & & 86 & 0.01 & 0.01 & & 43 & 0.01 & 0.01 & & 40 & 0.13 & 0.11 & \\
\hline \multirow{7}{*}{$\begin{array}{l}\text { Steering } \\
\text { Velocity }\end{array}$} & $\begin{array}{c}\text { No } \\
\text { Distraction }\end{array}$ & 78 & 0.02 & 0.01 & \multirow{7}{*}{3.75} & 156 & 0.04 & 0.03 & \multirow{7}{*}{0.94} & 78 & 0.02 & 0.01 & & 66 & 0.03 & 0.02 & \multirow{7}{*}{$3.04^{*}$} \\
\hline & $\begin{array}{c}\text { Hands-Free } \\
\text { Call }\end{array}$ & 81 & 0.02 & 0.01 & & 162 & 0.04 & 0.03 & & 81 & 0.02 & 0.01 & & 64 & 0.03 & 0.05 & \\
\hline & $\begin{array}{c}\text { Hand-Held } \\
\text { Call }\end{array}$ & 81 & 0.02 & 0.01 & & 160 & 0.04 & 0.03 & & 79 & 0.02 & 0.01 & & 70 & 0.02 & 0.02 & \\
\hline & $\begin{array}{c}\text { Voice } \\
\text { Commands } \\
\text { Text }\end{array}$ & 78 & 0.02 & 0.01 & & 154 & 0.04 & 0.04 & & 77 & 0.02 & 0.01 & $4.03^{*}$ & 69 & 0.02 & 0.02 & \\
\hline & Text & 77 & 0.02 & 0.01 & & 154 & 0.05 & 0.04 & & 77 & 0.02 & 0.01 & & 70 & 0.03 & 0.02 & \\
\hline & Clothing & 42 & 0.02 & 0.01 & & 84 & 0.06 & 0.04 & & 42 & 0.03 & 0.01 & & 38 & 0.03 & 0.01 & \\
\hline & $\begin{array}{l}\text { Eating or } \\
\text { Drinking }\end{array}$ & 43 & 0.02 & 0.01 & & 86 & 0.05 & 0.04 & & 43 & 0.02 & 0.01 & & 40 & 0.03 & 0.02 & \\
\hline
\end{tabular}




\begin{tabular}{|c|c|c|c|c|c|c|c|c|c|c|c|c|c|c|c|c|c|}
\hline \multicolumn{2}{|c|}{ Variables } & $\mathbf{N}$ & Mean & $\begin{array}{c}\text { Std. } \\
\text { Devia- } \\
\text { tion }\end{array}$ & $\mathbf{F}$ & $\mathbf{N}$ & Mean & $\begin{array}{c}\text { Std. } \\
\text { Devia- } \\
\text { tion }\end{array}$ & $\mathbf{F}$ & $\mathbf{N}$ & Mean & $\begin{array}{l}\text { Std. } \\
\text { Devia- } \\
\text { tion }\end{array}$ & $\mathbf{F}$ & $\mathbf{N}$ & Mean & $\begin{array}{c}\text { Std. } \\
\text { Devia- } \\
\text { tion }\end{array}$ & $\mathbf{F}$ \\
\hline \multirow{7}{*}{$\begin{array}{l}\text { Offset } \\
\text { From } \\
\text { Road } \\
\text { Center }\end{array}$} & $\begin{array}{c}\text { No } \\
\text { Distraction }\end{array}$ & 78 & 0.97 & 0.63 & \multirow{7}{*}{8.87} & 156 & 3.73 & 3.25 & \multirow{7}{*}{$6.27^{*}$} & 78 & 2.50 & 2.22 & \multirow{7}{*}{$4.43^{*}$} & 66 & 0.61 & 0.37 & \multirow{7}{*}{$5.97^{*}$} \\
\hline & $\begin{array}{c}\text { Hands-Free } \\
\text { Call }\end{array}$ & 81 & 0.96 & 0.65 & & 162 & 3.37 & 3.06 & & 81 & 2.48 & 2.34 & & 64 & 0.61 & 0.39 & \\
\hline & $\begin{array}{c}\text { Hand-Held } \\
\text { Call }\end{array}$ & 81 & 0.93 & 0.64 & & 160 & 3.53 & 3.11 & & 79 & 2.30 & 2.24 & & 70 & 0.61 & 0.39 & \\
\hline & $\begin{array}{c}\text { Voice } \\
\text { Commands } \\
\text { Text }\end{array}$ & 78 & 0.97 & 0.64 & & 154 & 3.59 & 3.23 & & 77 & 2.41 & 2.21 & & 69 & 0.58 & 0.33 & \\
\hline & Text & 77 & 0.95 & 0.62 & & 154 & 3.65 & 3.17 & & 77 & 2.36 & 2.21 & & 70 & 0.65 & 0.37 & \\
\hline & Clothing & 42 & 1.49 & 0.26 & & 84 & 6.28 & 1.76 & & 42 & 3.91 & 1.57 & & 38 & 0.90 & 0.31 & \\
\hline & $\begin{array}{l}\text { Eating or } \\
\text { Drinking }\end{array}$ & 43 & 1.53 & 0.27 & & 86 & 6.32 & 1.55 & & 43 & 3.81 & 1.61 & & 40 & 0.88 & 0.34 & \\
\hline \multirow{7}{*}{$\begin{array}{l}\text { Brake } \\
\text { Light }\end{array}$} & $\begin{array}{c}\text { No } \\
\text { Distraction }\end{array}$ & 78 & 0.09 & 0.46 & \multirow{7}{*}{1.68} & 156 & 0.26 & 0.98 & \multirow{7}{*}{$2.75^{*}$} & 78 & 0.60 & 1.42 & \multirow{7}{*}{2.37} & 66 & 2.32 & 3.06 & \multirow{7}{*}{$7.27^{*}$} \\
\hline & $\begin{array}{c}\text { Hands-Free } \\
\text { Call }\end{array}$ & 81 & 0.06 & 0.29 & & 162 & 0.35 & 1.14 & & 81 & 0.44 & 1.14 & & 64 & 2.08 & 2.97 & \\
\hline & $\begin{array}{c}\text { Hand-Held } \\
\text { Call }\end{array}$ & 81 & 0.23 & 0.69 & & 160 & 0.46 & 1.44 & & 79 & 0.65 & 1.72 & & 70 & 2.17 & 2.72 & \\
\hline & $\begin{array}{c}\text { Voice } \\
\text { Commands } \\
\text { Text }\end{array}$ & 78 & 0.10 & 0.44 & & 154 & 0.31 & 0.99 & & 77 & 0.35 & 1.12 & & 69 & 2.04 & 2.71 & \\
\hline & Text & 77 & 0.13 & 0.52 & & 154 & 0.27 & 0.97 & & 77 & 0.32 & 0.94 & & 70 & 2.23 & 2.98 & \\
\hline & Clothing & 42 & 0.00 & 0.00 & & 84 & 0.00 & 0.00 & & 42 & 0.00 & 0.00 & & 38 & 0.00 & 0.00 & \\
\hline & $\begin{array}{l}\text { Eating or } \\
\text { Drinking }\end{array}$ & 43 & 0.00 & 0.00 & & 86 & 0.00 & 0.00 & & 43 & 0.00 & 0.00 & & 40 & 0.00 & 0.00 & \\
\hline
\end{tabular}

Table 3 shows the result of the Post hoc Tukey, which reveals the significant difference of independent variables when comparing each type of distraction with no distraction. Steering velocity and brake did not change among different distractions. This result shows a negative relationship between eating/drinking and clothing distractions and deviation from the road center, probably due to taking their hands off the wheel to do so.

Participants significantly reduced their speed and throttle on all four road classes in all six distractions compared to the no-distraction scenario. Table 4 presents the speed change percentages between each distraction and no distraction for all four road classes.

As presented in Table 4 and Figure 5, the greatest speed reduction happened on rural and local roads, and clothing followed by eating/drinking had the highest speed reduction among all distractions. The results indicate that participants reduced their speeds almost the same percentages while distracted by a cell phone regardless of being hand-held or hands-free, which is consistent with some previous studies (Liu et al., 2019). 
Table 3

Post Hoc Tukey Analysis

\begin{tabular}{|c|c|c|c|c|c|c|c|c|c|c|}
\hline \multicolumn{3}{|c|}{ Variables } & $\begin{array}{c}\text { Mean } \\
\text { Difference } \\
(\mathrm{I}-\mathrm{J})\end{array}$ & $\begin{array}{l}\text { Std. } \\
\text { Error }\end{array}$ & $\begin{array}{c}\text { Mean } \\
\text { Difference } \\
(\mathrm{I}-\mathrm{J})\end{array}$ & $\begin{array}{l}\text { Std. } \\
\text { Error }\end{array}$ & \begin{tabular}{|c|} 
Mean \\
Difference \\
(I-J)
\end{tabular} & $\begin{array}{l}\text { Std. } \\
\text { Error }\end{array}$ & $\begin{array}{c}\text { Mean } \\
\text { Difference } \\
(\mathrm{I}-\mathrm{J})\end{array}$ & $\begin{array}{l}\text { Std. } \\
\text { Error }\end{array}$ \\
\hline \multirow{2}{*}{ DV } & \multirow{2}{*}{\multicolumn{2}{|c|}{ IV (Type of Distraction) }} & \multicolumn{8}{|c|}{ Type of Road } \\
\hline & & & \multicolumn{2}{|c|}{ Rural Collector } & \multicolumn{2}{|c|}{ Freeway } & \multicolumn{2}{|c|}{ Urban Arterial } & \multicolumn{2}{|c|}{ Local Road } \\
\hline \multirow{6}{*}{ Speed } & \multirow{6}{*}{$\begin{array}{c}\text { No } \\
\text { Distraction }\end{array}$} & Hands-Free Call & $14.28^{*}$ & 2.50 & $8.92^{*}$ & 0.89 & $7.37^{*}$ & 1.58 & $7.97^{*}$ & 1.84 \\
\hline & & Hand-Held Call & $15.58^{*}$ & 2.50 & $8.97^{*}$ & 0.90 & $6.88^{*}$ & 1.59 & $9.43^{*}$ & 1.80 \\
\hline & & Voice Commands Text & $16.25^{*}$ & 2.52 & $9.36^{*}$ & 0.90 & $8.90^{*}$ & 1.60 & $11.93^{*}$ & 1.81 \\
\hline & & Text & $14.53^{*}$ & 2.53 & $9.81^{*}$ & 0.90 & $7.05^{*}$ & 1.60 & $10.42^{*}$ & 1.80 \\
\hline & & Taking off or on Clothing & $20.69^{*}$ & 3.01 & $13.37^{*}$ & 1.08 & $7.88^{*}$ & 1.90 & $17.76^{*}$ & 2.13 \\
\hline & & Eating or Drinking & $14.96^{*}$ & 2.99 & $12.80^{*}$ & 1.07 & $10.02^{*}$ & 1.89 & $9.57^{*}$ & 2.10 \\
\hline \multirow{6}{*}{ Throttle } & \multirow{6}{*}{$\begin{array}{c}\text { No } \\
\text { Distraction }\end{array}$} & Hands-Free Call & $0.12^{*}$ & 0.02 & $0.04^{*}$ & 0.01 & 0.05 & 0.02 & 0.01 & 0.01 \\
\hline & & Hand-Held Call & $0.10^{*}$ & 0.02 & 0.04 & 0.01 & 0.04 & 0.02 & 0.00 & 0.01 \\
\hline & & Voice Commands Text & $0.11^{*}$ & 0.02 & $0.05^{*}$ & 0.01 & 0.04 & 0.02 & 0.02 & 0.01 \\
\hline & & Text & 0.04 & 0.02 & 0.04 & 0.01 & 0.01 & 0.02 & 0.01 & 0.01 \\
\hline & & Taking off or on Clothing & -0.01 & 0.03 & $0.07^{*}$ & 0.02 & 0.04 & 0.02 & 0.00 & 0.01 \\
\hline & & Eating or Drinking & -0.02 & 0.03 & $0.06^{*}$ & 0.01 & 0.02 & 0.02 & -0.01 & 0.01 \\
\hline \multirow{6}{*}{ Brake } & \multirow{6}{*}{$\begin{array}{c}\text { No } \\
\text { Distraction }\end{array}$} & Hands-Free Call & 0.00 & 0.00 & 0.00 & 0.00 & 0.00 & 0.00 & 0.01 & 0.01 \\
\hline & & Hand-Held Call & 0.00 & 0.00 & 0.00 & 0.00 & 0.00 & 0.00 & 0.01 & 0.01 \\
\hline & & Voice Commands Text & 0.00 & 0.00 & 0.00 & 0.00 & 0.00 & 0.00 & 0.01 & 0.01 \\
\hline & & Text & 0.00 & 0.00 & 0.00 & 0.00 & 0.00 & 0.00 & 0.01 & 0.01 \\
\hline & & Taking off or on Clothing & 0.00 & 0.00 & 0.00 & 0.00 & 0.00 & 0.00 & -0.02 & 0.02 \\
\hline & & Eating or Drinking & 0.00 & 0.00 & 0.00 & 0.00 & 0.00 & 0.00 & -0.03 & 0.02 \\
\hline \multirow{6}{*}{$\begin{array}{l}\text { Steering } \\
\text { Velocity }\end{array}$} & \multirow{6}{*}{$\begin{array}{c}\text { No } \\
\text { Distraction }\end{array}$} & Hands-Free Call & 0.00 & 0.00 & 0.00 & 0.00 & 0.00 & 0.00 & 0.00 & 0.00 \\
\hline & & Hand-Held Call & 0.00 & 0.00 & 0.00 & 0.00 & 0.00 & 0.00 & 0.00 & 0.00 \\
\hline & & Voice Commands Text & 0.00 & 0.00 & -0.01 & 0.00 & 0.00 & 0.00 & 0.01 & 0.00 \\
\hline & & Text & 0.00 & 0.00 & $-0.01^{*}$ & 0.00 & 0.00 & 0.00 & 0.00 & 0.00 \\
\hline & & Taking off or on Clothing & 0.00 & 0.00 & $-0.02^{*}$ & 0.00 & $-0.01^{*}$ & 0.00 & 0.00 & 0.00 \\
\hline & & Eating or Drinking & 0.00 & 0.00 & $-0.02^{*}$ & 0.00 & 0.00 & 0.00 & -0.01 & 0.00 \\
\hline \multirow{6}{*}{$\begin{array}{l}\text { Offset } \\
\text { from } \\
\text { Road } \\
\text { Center }\end{array}$} & \multirow{6}{*}{$\begin{array}{c}\text { No } \\
\text { Distraction }\end{array}$} & Hands-Free Call & 0.01 & 0.09 & 0.36 & 0.33 & 0.01 & 0.35 & 0.00 & 0.06 \\
\hline & & Hand-Held Call & 0.05 & 0.09 & 0.20 & 0.34 & 0.19 & 0.35 & 0.00 & 0.06 \\
\hline & & Voice Commands Text & 0.00 & 0.10 & 0.14 & 0.34 & 0.09 & 0.35 & 0.04 & 0.06 \\
\hline & & Text & 0.03 & 0.10 & 0.08 & 0.34 & 0.14 & 0.35 & -0.03 & 0.06 \\
\hline & & Taking off or on Clothing & $-0.52^{*}$ & 0.11 & $-2.55^{*}$ & 0.40 & $-1.41^{*}$ & 0.42 & $-0.29^{*}$ & 0.07 \\
\hline & & Eating or Drinking & $-0.55^{*}$ & 0.11 & $-2.58^{*}$ & 0.40 & $-1.31^{*}$ & 0.42 & $-0.26^{*}$ & 0.07 \\
\hline \multirow{6}{*}{$\begin{array}{l}\text { Brake } \\
\text { Light }\end{array}$} & \multirow{6}{*}{$\begin{array}{c}\text { No } \\
\text { Distraction }\end{array}$} & Hands-Free Call & 0.03 & 0.09 & -0.09 & 0.12 & 0.16 & 0.19 & 0.24 & 0.48 \\
\hline & & Hand-Held Call & -0.14 & 0.09 & -0.19 & 0.12 & -0.04 & 0.19 & 0.15 & 0.47 \\
\hline & & Voice Commands Text & -0.01 & 0.09 & -0.05 & 0.12 & 0.25 & 0.19 & 0.27 & 0.47 \\
\hline & & Text & -0.04 & 0.09 & -0.01 & 0.12 & 0.28 & 0.19 & 0.09 & 0.47 \\
\hline & & Taking off or on Clothing & 0.09 & 0.10 & $0.26^{*}$ & 0.14 & 0.60 & 0.23 & $2.32^{*}$ & 0.55 \\
\hline & & Eating or Drinking & 0.09 & 0.10 & $0.26^{*}$ & 0.14 & 0.60 & 0.23 & $2.32^{*}$ & 0.55 \\
\hline
\end{tabular}


Table 4

Speed Change in Comparison with No Distraction

\begin{tabular}{|c|c|c|c|c|}
\hline Type of Road & $\begin{array}{c}\text { Rural } \\
\text { Collector }\end{array}$ & Freeway & Urban Arterial & $\begin{array}{c}\text { Local Road in a } \\
\text { School Zone }\end{array}$ \\
\hline Hands-Free Call & $-21 \%$ & $-11 \%$ & $-14 \%$ & $-22 \%$ \\
\hline Hand-Held Call & $-22 \%$ & $-11 \%$ & $-13 \%$ & $-26 \%$ \\
\hline Voice Commands Text & $-19 \%$ & $-11 \%$ & $-17 \%$ & $-33 \%$ \\
\hline Text & $-15 \%$ & $-12 \%$ & $-14 \%$ & $-29 \%$ \\
\hline Taking off or on Clothing & $-24 \%$ & $-16 \%$ & $-15 \%$ & $-50 \%$ \\
\hline
\end{tabular}

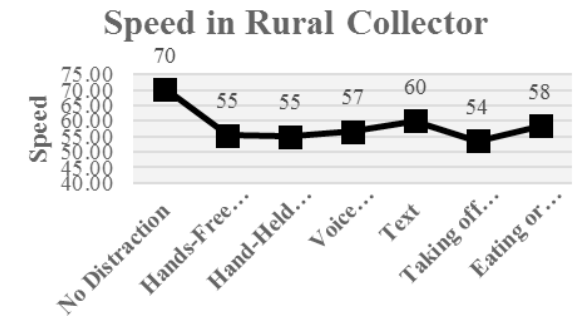

Types of Distraction
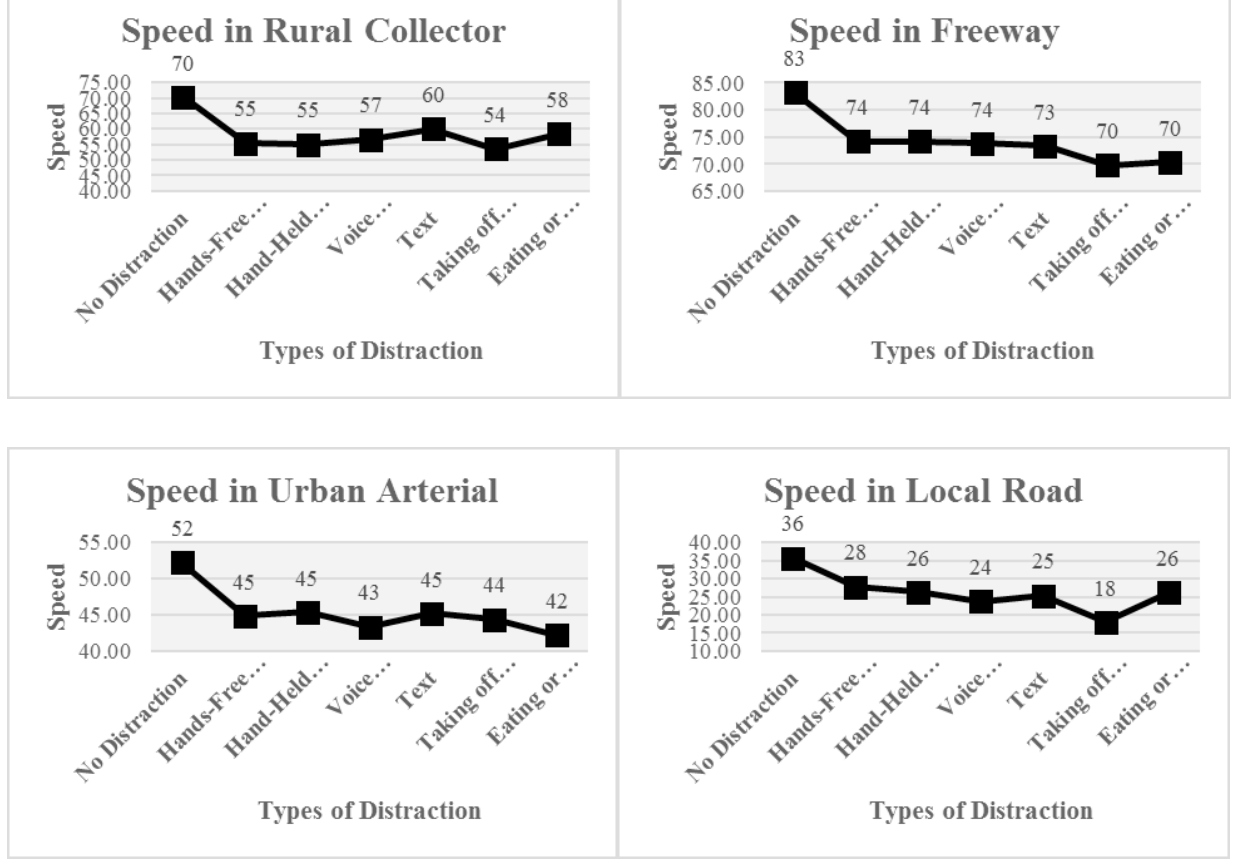

Fig. 5.

Speed Variations Among Various Distractions on Different Road Classes 
This study found clothing eating/drinking is highly distractive. Participants deviated from the lane center and reduced their speed tremendously while taking their clothes on/ off and eating/drinking.

\section{Conclusions}

This study investigates the effect of six different distractions on drivers' behavior using a driving simulator. Some 92 participants drove one base scenario (without distraction) and six distraction scenarios; each scenario took about 15 minutes with different types of distraction including no cell phone, hands-free call, hand-held call, voice commands text, text, clothing, eating and drinking. The results showed that participants decreased their speed in the presence of all cell phone-related distractions on all roads. Furthermore, speed reduction was the highest when distracted by clothing and eating/drinking. The results suggest a full ban on cell phone usage, not just hand-held devices. Also, transportation safety policymakers may need to regulate clothing and eating/drinking. The highest speed reduction happened on the local road when clothing (50\%), voice command texting (33\%), and texting (29\%). In general, speed reduction was the highest on the local road probably because of high cognitive load (stop signs and traffic lights, pedestrians and cyclists). The high-speed reduction on the rural road was partly because of driving way over the speed limit due to low traffic and very few intersections. This could lead to crashes when high-speed vehicles approach the distracted low-speed vehicle, or an animal or a pedestrian cross those roads.

\section{Acknowledgments}

The authors would like to thank the Maryland Department of Transportation-
Motor Vehicle Administration-Maryland Highway Safety Office (GN-Morgan State -2019-291) and the Urban Mobility \& Equity Center, a Tier 1 University Transportation Center of the U.S. DOT University Transportation Centers Program, at Morgan State University for their funding support.

\section{References}

Ahangari, S.; Hasanpour, A.H.; Khadem, N.; Banerjee, S. 2019. Investigating the Impact of Distracted Driving among Different Socio-Demographic Groups. Urban Mobility \& Equity Center, Morgan State University, Baltimore, 54p.

Charlton, S. G. 2009. Driving while conversing: Cell phones that distract and passengers who react, Accident Analysis \& Prevention 41(1): 160-173.

Choudhary, P.; Velaga, N. R. 2017. Analysis of vehiclebased lateral performance measures during distracted driving due to phone use, Transportation Research Part F: Traffic Psychology and Behaviour 44: 120-133.

Drews, F. A.; Pasupathi, M.; Strayer, D. L. 2004. Passenger and cell-phone conversations in simulated driving, Proceedings of the Human Factors and Ergonomics Society Annual Meeting 48: 2210-2212.

Drews, F. A.; Yazdani, H.; Godfrey, C. N.; Cooper, J. M.; Strayer, D. L. 2009. Text messaging during simulated driving, Human Factors 51(5): 762-770.

Gliklich, E.; Guo, R.; Bergmark, R. W. 2016. Texting while driving: A study of 1211 US adults with the Distracted Driving Survey, Preventive Medicine Reports 4: 486-489.

He, J.; Chaparro, A.; Nguyen, B.; Burge, R. J.; Crandall, J.; Chaparro, B.; Ni, R.; Cao, S. 2014. Texting while driving: Is speech-based text entry less risky than handheld text entry?, Accident Analysis \& Prevention 72: 287-295. 
Horberry, T.; Anderson, J.; Regan, M. A.; Triggs, T. J.; Brown, J. 2006. Driver distraction: The effects of concurrent in-vehicle tasks, road environment complexity and age on driving performance, Accident Analysis \& Prevention 38(1): 185-191.

Lee, J. D. 2017. Driver Distraction and Inattention: Advances in Research and Countermeasures, CRCPress Vol. 1.

Lee, J. Y.; Lee, J. D.; Bärgman, J.; Lee, J.; Reimer, B. 2018. How safe is tuning a radio?: Using the radio tuning task as a benchmark for distracted driving, Accident Analysis \& Prevention 110: 29-37.

Lipovac, K.; Djerić, M.; Tešić, M.; Andrić, Z.; Marić, B. 2017. Mobile phone use while driving-literary review, Transportation Research Part F: Traffic Psychology and Behaviour 47: 132-142.

Liu, C.; Lu, C.; Wang, S.; Sharma, A.; Shaw, J. 2019. A longitudinal analysis of the effectiveness of California's ban on cellphone use while driving, Transportation Research Part A: Policy and Practice 124: 456-467.

Neale, V. L.; Dingus, T. A.; Klauer, S. G.; Sudweeks, J.; Goodman, M. 2005. An overview of the 100-carnaturalistic study and findings, National Highway Traffic Safety Administration, 05-0400: 1-10

NHTSA. 2015. National Survey on Distracted Driving Attitudes and Behaviors, Behavioral Safety Research. Available from Internet: <https://rosap.ntl.bts.gov/ view/dot/35960>.

Overton, T. L.; Rives, T. E.; Hecht, C.; Shafi, S.; Gandhi, R. R. 2015. Distracted driving: Prevalence, problems, and prevention, International Journal of Injury Control and Safety Promotion 22(3): 187-192.
Oviedo-Trespalacios, O.; Williamson, A.; King, M. 2019. User preferences and design recommendations for voluntary smartphone applications to prevent distracted driving, Transportation Research Part F: Traffic Psychology and Behaviour 64: 47-57.

Schlehofer, M. M.; Thompson, S. C.; Ting, S.; Ostermann; S.; Nierman, A.; Skenderian, J. 2010. Psychological predictors of college students' cell phone use while driving, Accident Analysis \& Prevention 42(4): 1107-1112.

Stavrinos, D.; Jones, J. L.; Garner, A. A.; Griffin, R.; Franklin, C. A.; Ball, D.; Welburn, S. C.; Ball, K. K.; Sisiopiku, V. P.; Fine, P. R. 2013. Impact of distracted driving on safety and traffic flow, Accident Analysis \& Prevention 61: 63-70.

Stutts, J. C.; Reinfurt, D. W.; Rodgman, E. A. 2001. The role of driver distraction in crashes: An analysis of 1995-1999 Crashworthiness Data System Data. In Annual Proceedings. Association for the Advancement of Automotive Medicine, 45: 287-301.

Thomas, S.; Zuckman, J.; Beck, D. 2012. AT\&T teen driver survey. Available from internet: <https://www. att.com/Common/about_us/txting_driving/att_teen survey_executive.pdf $>$.

Törnros, J. E.; Bolling, A. K. 2005. Mobile phone use-Effects of handheld and handsfree phones on driving performance, Accident Analysis \& Prevention 37(5): 902-909.

\section{jitte 265}

